\title{
5' Exon replacement and repair by spliceosome-mediated RNA trans-splicing
}

\author{
S. GARY MANSFIELD, ${ }^{1}$ REBECCA HAWKINS CLARK, ${ }^{1}$ M. PUTTARAJU, ${ }^{1}$ JOLANTA KOLE, ${ }^{2}$ \\ JONATHAN A. COHN, ${ }^{2}$ LLOYD G. MITCHELL, $^{1}$ and MARIANO A. GARCIA-BLANCO ${ }^{1}$ \\ ${ }^{1}$ Intronn, Inc., Gaithersburg, Maryland 20878, USA \\ ${ }^{2}$ Department of Medicine, Duke University Medical Center, Durham, North Carolina 27713, USA
}

\begin{abstract}
Spliceosome-mediated RNA trans-splicing (SMaRT) has been used previously to reprogram mutant endogenous CFTR and factor VIII mRNAs in human epithelial cell and tissue models and knockout mice, respectively. Those studies used 3' exon replacement (3'ER); a process in which the distal portion of RNA is reprogrammed. Here, we also show that the $5^{\prime}$ end of mRNA can be completely rewritten by 5'ER. For proof-of-concept, and to test whether 5'ER could generate functional CFTR, we generated a mutant minigene target containing CFTR exons 10-24 $(\Delta \mathrm{F} 508)$ and a mini-intron 10, and a pretrans-splicing molecule (targeted to intron 10) containing CFTR exons 1-10 (+F508), and tested these two constructs in 293T cells for anion efflux transport. Cells cotransfected with target and PTM showed a consistent increase in anion efflux, but there was no response in control cells that received PTM or target alone. Using a LacZ reporter system to accurately quantify trans-splicing efficiency, we tested several unique PTM designs. These studies provided two important findings as follows: (1) efficient trans-splicing can be achieved by binding the PTM to different locations in the target, and (2) relatively few changes in PTM design can have a profound impact on trans-splicing activity. Tethering the PTM close to the target $3^{\prime}$ splice site (as opposed to the donor site) and inserting an intron in the PTM coding resulted in a 65-fold enhancement of LacZ activity. These studies demonstrate that (1) SMaRT can be used to reprogram the $5^{\prime}$ end of mRNA, and (2) efficiency can be improved substantially.
\end{abstract}

Keywords: CFTR; genetic disease; mRNA repair; SMaRT, trans-splicing

\section{INTRODUCTION}

Many recent developments underscore the importance of RNA as a tool and a target in biomedical applications (Sullenger and Gilboa 2002). The application of RNA interference to efficiently and specifically knockdown expression of mammalian gene products has opened a novel avenue for experimental and therapeutic applications designed to reduce levels of an undesirable protein (Shi 2003). From a therapeutic standpoint, it is equally important to recover activities lost in disease states or reprogram mRNA to express a completely different protein. A second RNA technology, mRNA reprogramming by trans-splicing, offers the ability to repair mRNAs, and thus, proteins.

Reprint requests to: S. Gary Mansfield, Intronn, Inc., 910 Clopper Road, South Building, Suite 210, Gaithersburg, MD 20878, USA; e-mail: gmansfield@intronn.com; fax: (240)683-2451.

Abbreviations: $\mathrm{BD}$, binding domain; CFTR, cystic fibrosis transmembrane conductance regulator; $5^{\prime} \mathrm{ER}, 5^{\prime}$ exon replacement; $3^{\prime} \mathrm{ER}, 3^{\prime}$ exon replacement; TSD, trans-splicing domain.

Article and publication are at http://www.rnajournal.org/cgi/doi/ 10.1261/rna.5101903.
RNA reprogramming has been achieved using group I trans-splicing ribozymes and by spliceosome-mediated RNA trans-splicing (SMaRT). SMaRT is a unique technology with many proven and potential applications (GarciaBlanco et al. 2000). Recently, we demonstrated the feasibility of using SMaRT to repair endogenous cystic fibrosis (CF) mRNAs encoding the cystic fibrosis transmembrane conductance regulator (CFTR) in human epithelial cells in culture and in a xenograft model (Mansfield et al. 2000; Liu et al. 2002), and factor VIII mRNA in hemophilia A knockout mice (Chao et al. 2003), using $3^{\prime}$ exon replacement ( $\left.3^{\prime} \mathrm{ER}\right)$. During $3^{\prime} \mathrm{ER}$, the distal portion of a target mRNA is completely rewritten by trans-splicing sequences between the target pre-mRNA and a repair construct called a pretrans-splicing molecule (PTM) (Puttaraju et al. 1999, 2001). To effect CFTR 3' end repair, a PTM was designed to base pair with the $3^{\prime}$ splice site of intron 9 and trans-splice the normal coding sequences of exons 10-24 (in which exon 10 of the target carries the CF $\Delta$ F508 deletion) (Mansfield et al. 2000; Liu et al. 2002). 3'ER PTMs are composed of several modular domains that include binding-domain sequences 
that are complementary to the target pre-mRNA, a spacer sequence, and a 3 ' splice site followed by virtually any coding sequence. The $3^{\prime}$ splice site typically includes a branchpoint consensus sequence, polypyrimidine tract, and a splice acceptor AG dinucleotide. We term these latter three elements together with the binding domain and spacer sequence, the trans-splicing domain (TSD). This region constitutes the active site of the PTM, because it contains the sequence elements that are necessary for efficient transsplicing. All of the PTM domains described above can be changed to alter the efficiency and specificity of SMaRT (Puttaraju et al. 1999, 2001; Mansfield et al. 2000).

The strategy of mRNA correction offers several potential advantages, such as reduced transgene size, elimination of ectopic expression of the repaired product, the acquisition of endogenous regulation, and the ability to include large regulatory elements in vectors with limited packaging capacity. The need to repair mutations throughout an mRNA and the versatility of SMaRT technology led us to perform experiments designed to reprogram the $5^{\prime}$ region of mRNA by $5^{\prime}$ exon replacement ( $\left.5^{\prime} \mathrm{ER}\right)$. The consensus sequence that defines the $5^{\prime}$ donor site, AG/GURAGU, is less extensive than the $3^{\prime}$ splice elements (Moore et al. 1999), and it has remained uncertain whether this site could be efficiently exploited to effect trans-splicing repair. Group I ribozymes have been used to perform trans-splicing reactions in vitro to repair globin mRNA in sickle cell anemia-derived erythroid precursor cells (Lan et al. 1998), and trans-splicing ribozymes have also been used successfully to induce changes in p53 and chloride channels (Watanabe and Sullenger 2000; Rogers et al. 2002) by a 3' replacement process; to our knowledge, there is no published report demonstrating that ribozymes are capable of repair in a $5^{\prime}$ manner. Additionally, in trypanosomes (Van der Ploeg 1986) and Caenorhabditis elegans (Krause and Hirsh 1987), it has been demonstrated that RNAs are commonly modified by the addition of a splice leader on the $5^{\prime}$ terminus by transsplicing. This process is different from SMaRT in that the latter does not represent a replacement of sequences between the target and modifying molecule. In the present study, our key questions were as follows: (1) whether the 5' end of mRNA could be efficiently reprogrammed; (2) how the efficiency of the process could be optimized; and (3) which regions of a pre-mRNA, that is, exon, intron, splice junction, or a combination of these, could be targeted to increase trans-splicing. The ability to reprogram the $5^{\prime}$ region of mRNAs confers a unique advantage to SMaRT that is particularly relevant for diseases in which the mutation(s) are $5^{\prime}$ proximal in the RNA.

Using CFTR as a model of a genetic disease, and a $\beta$-galactosidase repair model to quantify levels of functional restoration, we demonstrate here that PTMs can be engineered to repair mutant mRNA by $5^{\prime} \mathrm{ER}$. This work is unique because, to date, alteration of RNA or DNA has focused on 3' ER. We show that trans-splicing is precise and can replace coding sequences of at least $1.9 \mathrm{~kb}$, and that the efficiency of 5'ER can be increased many fold by targeting different regions of pre-mRNA. This advancement supports broader applications of SMaRT technology and suggests that regardless of position within the transcript, any codon can be corrected or any sequence modified by substitution.

\section{RESULTS}

\section{SMaRT modification of mRNA by $5^{\prime}$ exon replacement}

Previous work to date has focused primarily on PTMs that utilize a $3^{\prime}$ splice site and have been termed 3'ER PTMs (Puttaraju et al. 1999, 2001; Mansfield et al. 2000). To test the efficiency of repair by $5^{\prime} \mathrm{ER}$, we developed a mutant $\Delta$ F508 CFTR minigene target and a 5'ER PTM for repairing the target mRNA. This PTM, CFTR-PTM11, contained the normal human CFTR-coding sequences for exons 1-10 (including codon F508 in exon 10), a TSD consisting of a $5^{\prime}$ donor site (AG/GUAAGA), a spacer sequence, and a binding domain of 31 bases complementary to the target. The first binding domain was designed to bind the exon 10intron 10 junction of the target, with the intention of blocking cis-splicing. The target, CFTR-T11, consisted of mutant exon $10\left(\Delta\right.$ F508), a mini-intron 10 containing the $5^{\prime}$ and $3^{\prime}$ ends of intron 10 from the human CFTR gene, and the coding sequence for exons 11 through 24 (Fig. 1A). The repair process for $5^{\prime} \mathrm{ER}$ is illustrated in Figure $1 \mathrm{~A}$.

CFTR-PTM11 and CFTR-T11 were cotransfected into human embryonic kidney 293T cells (293T), the cells were harvested $48 \mathrm{~h}$ after transfection, and total RNA was isolated. Trans- and cis-spliced RNA products were detected by RT-PCR using primers that would specifically amplify cis(CF1 and CF111) and trans-spliced (CF93 and CF111) products. In those samples that received PTM, target, or pc3.1DNA(-) vector alone, or PTM plus target, but with no reverse transcription, no PCR products were detected. However, in the sample that received PTM and target, a specific product of 265 bp was generated, which is the predicted size for a trans-spliced product between the PTM and target (Fig. 1B). Sequencing of these presumptive cis- and trans-spliced cDNAs confirmed (1) the identity of each product (data not shown); (2) that trans-splicing took place at the intended splice sites; and (3) that the repaired product contained modified codons in exon 10. These three observations demonstrate that the repaired product was generated by trans-splicing between the target and PTM. Semiquantitative RT-PCR assays using primers that were specific for cis- and trans-spliced mRNAs showed that the level of trans-splicing was $\sim 6 \%$ of target pre-mRNA cissplicing (data not shown). These results clearly show that trans-splicing can be used to efficiently reprogram the $5^{\prime}$ end of mRNA by the substitution of target sequences with PTM sequences. 
A.
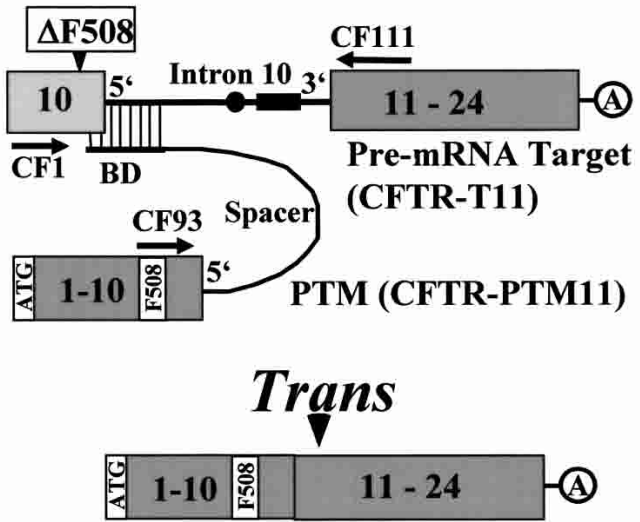

Repaired full length CFTR mRNA

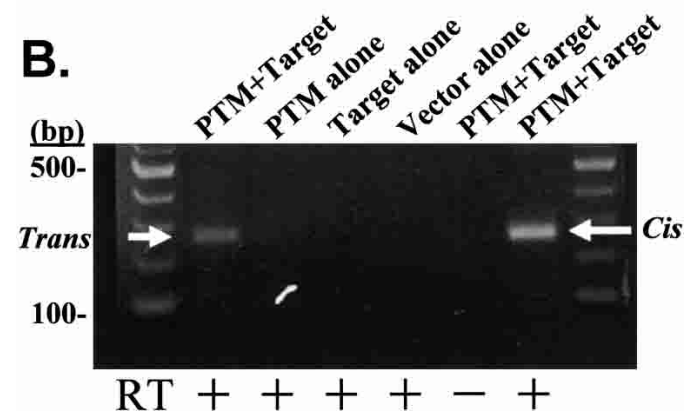

FIGURE 1. CFTR model constructs and illustration of trans-splicing by $5^{\prime}$ exon replacement. (A) Detailed structure of a 5'ER PTM (CFTRPTM11), and a mini-gene target (CFTR-T11). The PTM lacks a poly(A) signal and the target lacks a methionine initiator codon. Modified codons are engineered in exons 9 and 10 of the PTM, and exons 11 and 12 of the target to aid in distinguishing trans-spliced products from cis-spliced or endogenous CFTR products. The position of the three oligonucleotide primers (CF1, CF93, and CF111) used in RT-PCR experiments are indicated by arrows. The diagram shows the PTM binding to the $5^{\prime}$ splice site of intron 10 of the minigene target at the pre-mRNA level. $(B)$ Demonstration of trans-splicing by RTPCR. Cells were transfected with either PTM plus target, or PTM, target, or vector (pc3.1DNA) alone, or PTM plus target, but without the reverse transcription step (four different negative controls). Cisspliced $(\Delta \mathrm{F} 508)$ products were detected with primers CF1 and CF111 (right arrow), and trans-spliced products with primers CF93 and CF111 (left arrow). (BD) Binding domain.

\section{Repair of CFTR $\Delta$ F508 pre-mRNA by $5^{\prime}$ ER produces functional ion channels in cells}

The next goal of this study was to demonstrate that transsplicing by $5^{\prime}$ ER can produce copies of CFTR that are functional as ion transporters at the cell membrane. CFTR is a transmembrane protein that regulates the flow of chloride ion across the cell membrane in epithelial cells in a cAMPdependent fashion. For this experiment, we used a CFTRbased PTM (CFTR-PTM30), with a binding domain complementary to exon 10 and its splice sites. Anion efflux was compared in 293T cells transfected with target only (CFTR-T11), PTM only (CFTR-PTM30), or with both. Un- der baseline conditions, anion efflux was equivalent under all three conditions. Following forskolin and IBMX treatment of 293T monolayers (which is predicted to elevate cAMP levels and stimulate cAMP-dependent ion channel activity), there was a consistent increase in anion efflux transport response in cells cotransfected with target plus PTM. In contrast, there was no response in cells transfected with target alone and only a minor response in cells receiving PTM alone (arrows, Fig. 2). It is unclear why values for target and PTM alone are not identical, although similar results were obtained for 3'ER CFTR PTMs (S.G. Mansfield, R.H. Clark, M. Puttaraju, L.G. Mitchell, and M.A. Garcia-Blanco, unpubl.). These results suggest that fulllength repaired mRNA is generated by trans-splicing and that SMaRT is capable of generating functional CFTR ion channels in cells.

\section{5 ' Exon replacement efficiency can be increased significantly}

For 3'ER, we had demonstrated previously that increasing the binding domain length from 23 to 153 nucleotides gave much higher trans-splicing efficiency (Puttaraju et al. 2001). To assess how the efficiency of $5^{\prime} \mathrm{ER}$ can be optimized, we developed a mutant LacZ-CFTR repair model consisting of the $L a c Z$ coding unit (minus 124 bases) split into two exons

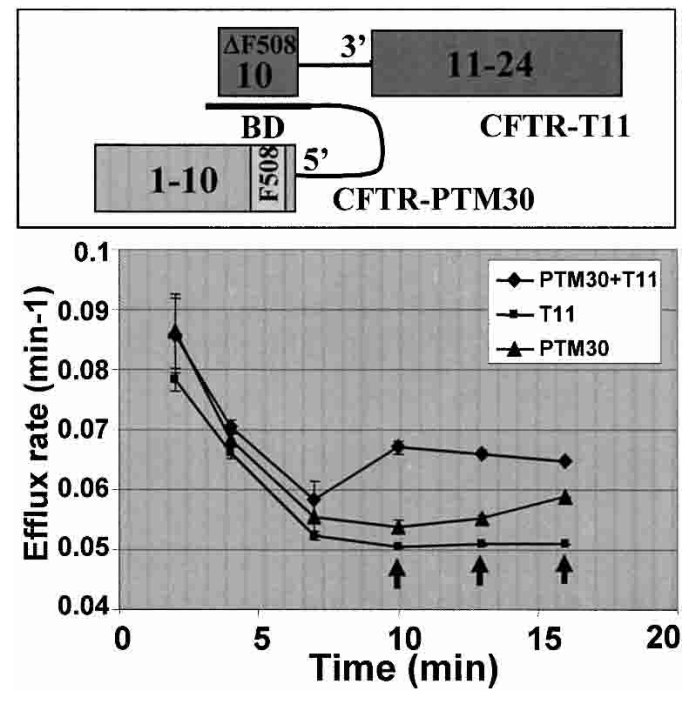

FIGURE 2. RNA trans-splicing by $5^{\prime}$ exon replacement generates functional ion channels in human cells. HEK 293T cells were transfected with either target alone (CFTR-T11), PTM alone (CFTRPTM30), or with target and PTM. Cell populations were incubated in medium containing ${ }^{125}$ Iodine, and then tested for the presence of ion channel efflux activity (in $\mathrm{min}^{-1}$ ) by stimulation with forskolin and IBMX at intervals of $3 \mathrm{~min}$ (time points 7, 10, 13, and $16 \mathrm{~min}$ ). Forskolin is predicted to elevate cAMP levels and stimulate cAMPdependant ion channel activity. Only those monolayers that received a PTM and target showed substantial increases in anion efflux at three time-points (arrows). Values represent mean \pm standard error of two independent transfections, with the exception of the 4-min time point for PTM plus target, which represents a single data point. (BD) Binding domain. 
of 1788 and $1256 \mathrm{bp}$, and separated by CFTR mini-introns 9 and 10, and a mutant exon 10 ( $\Delta$ F508) (Fig. 3A, LacZCF10m). We engineered PTMs encoding the $5^{\prime}$ portion of LacZ (1912 bp) (Fig. 3A) and a binding domain complementary to different regions of the LacZ-CFTR target (Fig. 3B). Cis-splicing of all exons or just the two LacZ exons (Ibid.) (through exon skipping) does not encode functional $\beta$-galactosidase. However, accurate 5'ER transsplicing between the target and a LacZ PTM is predicted to produce a functional $L a c Z$ mRNA (Fig. 3A). To test whether binding different areas of the target could result in increased trans-splicing, we generated five different PTMs with binding domains that would occlude either the $3^{\prime}$ splice site of intron 9 , the $5^{\prime}$ splice site of intron 10 , exon 10 , or a com-

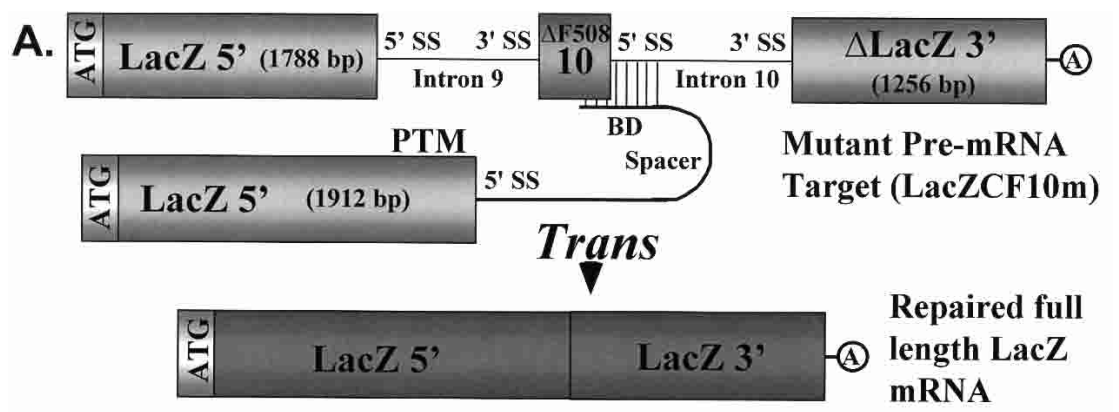

B.
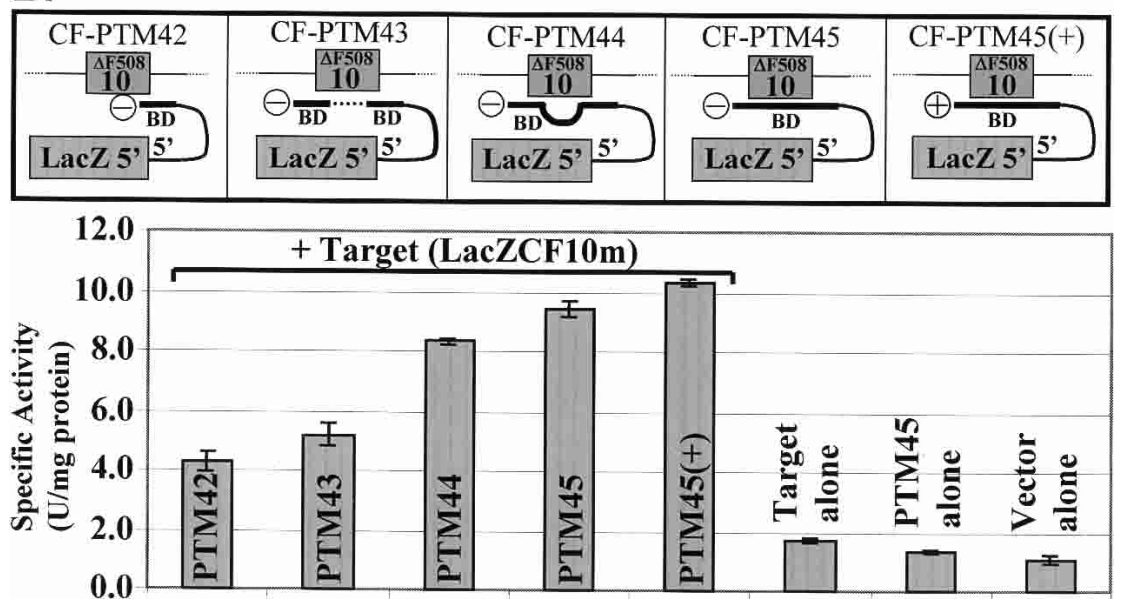

FIGURE 3. Model system to optimize trans-splicing efficiency. (A) Schematic diagram showing a LacZ PTM binding to a chimeric LacZ- $\Delta$ F508-CFTR target at the pre-mRNA level. $(B)$ Trans-splicing efficiency by $5^{\prime}$ exon replacement is increased by binding to more than one splice site. The panels above the histogram shows the location of four different PTM-binding domains (CF-PTM42, CF-PTM43, CF-PTM44, CF-PTM45) targeted to different regions of a defective LacZ- $\Delta$ F508 target (LacZCF10m). CF-PTM45 and CF-PTM45(+) are identical, except that the latter has a poly(A) signal and the former does not. The histogram below the five panels compares the repair efficiency of the five PTMs using an in-solution $\beta$-galactosidase assay. Transfections, preparation of cell lysates, and assessment of activity was as described previously (Puttaraju et al. 2001). Values are the mean of two independent experiments $( \pm$ standard error) and are expressed as units of $\beta$-galactosidase activity per milligram of total protein. PTMs and the target are cloned in pc3.1DNA(-). The vector-alone sample refers to plasmid pc3.1DNA(-) that does not contain either a PTM or a target. Each transfection was performed with $2 \mu \mathrm{g}$ of target plasmid and $2 \mu \mathrm{g}$ of PTM plasmid. All samples contained the same amount of plasmid DNA; PTM-alone and target-alone samples were balanced to $4 \mu \mathrm{g}$ with vector pc3.1DNA(-). (BD) Binding domain; (SS) splice site; (+), with bGH poly(A) signal; (-), without bGH polyA signal. bination of these (Fig. 3B). The binding domain of CFPTM42 covered the $5^{\prime}$ splice site of intron 10. CF-PTM43 had a binding domain complementary to noncontiguous sequence in the target, such that it would bind to the $3^{\prime}$ and 5 splice sites of introns 9 and 10, respectively. CF-PTM44 was similar to CF-PTM43, except that we included a 140-bp sequence (nonbinding) between the two sequence mone (bGH) poly(A) signal to reduce the possibility of plasmic transport and translation in the absence of trans-splicing. CF-PTM45+ has a poly(A) signal, and was constructed to compare how the presence or absence of this signal affects transsplicing. Each PTM was transiently cotransfected into 293T cells with target LacZCF10m, and cell lysates were assayed for $\beta$-galactosidase activity after $48 \mathrm{~h}$. These experiments showed that occluding the competing exon 10 can significantly enhance trans-splicing efficiency (2.2-fold over the first design; compare CF-PTM42 and CF-PTM45 in Fig. 3B), although an effect due to size of the binding domain cannot be formally excluded. The inclusion of a bGH poly(A) site slightly enhanced the transsplicing efficiency. Additional experiments testing whether an alpha mosaic virus translational enhancer sequence (Jobling and Gehrke 1987) and a consensus Kozak sequence around the ATG would enhance expression of the mRNA did not show the expected effect, indeed, these modifications caused a slight decrease in activity (data not shown). Finally, in another experiment in which CF-PTM45 was engineered with a cis-acting hammerhead ribozyme (capable of self-cleavage) (Chowrira et al. 1994) with the intention of generating a poly (A) minus PTM with a precise $3^{\prime}$ end, we did not observe any enhancement of trans-splicing (data not shown).

To substantiate the results from the in-solution $\beta$-galactosidase assays, we also performed in situ staining with several cell populations that had either received PTM and target, PTM alone, or target alone. Cells stained strongly posi- 
tive for $\beta$-galactosidase only in samples that had received a PTM and a target (data not shown). The first series of PTMs described above demonstrate that mRNA can be rewritten at its $5^{\prime}$ end by SMaRT, the process is precise and the efficiency can be altered by binding at different regions in the target, but the process is relatively inefficient using these particular PTMs.

\section{PTMs targeted closer to the target acceptor site produce a significant increase in efficiency}

The first set of 5'ER designs (CF-PTM42 to CF-PTM45) for this particular study were made to occlude the $5^{\prime}$ and/or $3^{\prime}$ splice site of CFTR mini-intron 10 and suppress cis-splicing. This theory of blocking the cis-splicing elements had been first tested in 3'ER PTMs, in which binding domains were developed to occlude the branchpoint, polypyrimidine tract, and acceptor site of the target intron. However, more recent work has shown clearly that this design feature is not essential for effective trans-splicing, and that other regions of an intron can be targeted (S.A. Mansfield, P. Du, S. Hiriyanna, R.C. Bartel, and E. Otto, unpubl.). We hypothesized that targeting the $5^{\prime}$ ER PTM close to the 3 ' end of the target intron would bring the PTM donor site in close proximity to the target acceptor site, but without sterically occluding the $3^{\prime}$ splice site (required for trans-splicing). CFPTM50 was re-engineered with a binding domain of 125 nucleotides complementary to sequence just upstream (position -62 to -186$)$ of the intron 9 predicted branchpoint. The final construct was termed CF-PTM53 (Fig. 4). In these constructs, the length of sequence between the donor site and binding domain (referred to as a spacer sequence in 3'ER constructs; Puttaraju et al. 1999) was shortened from 60 to 10 bases, firstly to assess the effect of the spacer on
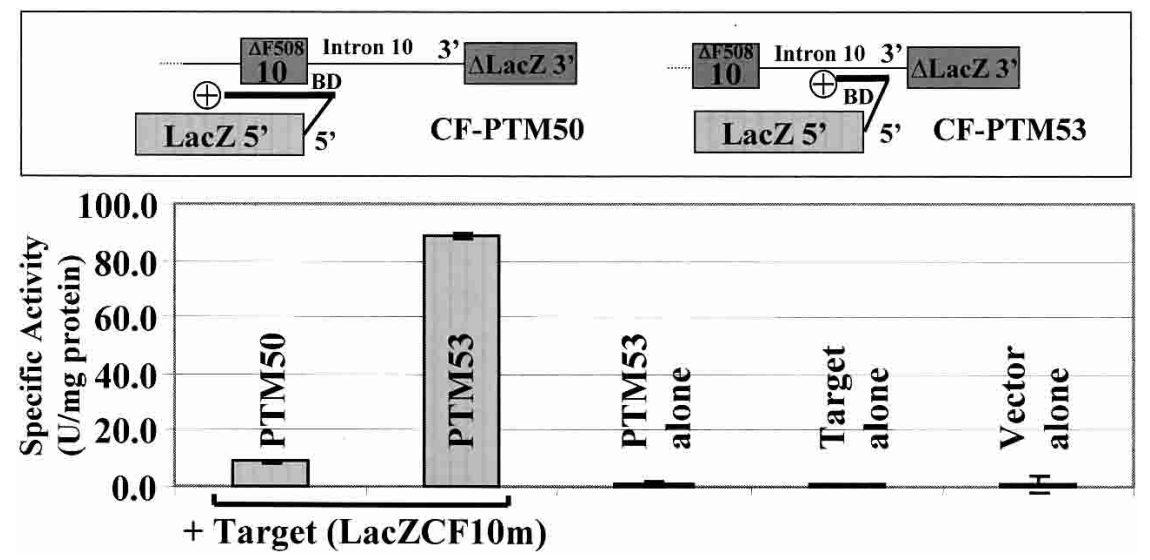

FIGURE 4. Targeting the PTM closer to the intended $3^{\prime}$ splice site results in a substantial increase in trans-splicing efficiency. The panel above the histogram shows a schematic of two different PTMs and the location of their binding domain with respect to the target acceptor site. The binding domain of CF-PTM53 is located $\sim 400$ bases downstream of CF-PTM50. Both PTMs have a spacer sequence that is shorter than all previous constructs by 50 bases. Values are mean \pm standard error of three separate experiments. (BD) Binding domain.
PTM activity, and secondly to ensure that the PTM donor and target acceptor were in close proximity. Both constructs are identical with the exception of the binding domain position and size. Retargeting the PTM in this manner resulted in a 10-fold increase in trans-splicing (Fig. 4). Thus, although the PTMs differ in their binding-domain size, these data suggest that approximating the PTM near the $3^{\prime}$ splice site enhances the efficiency of trans-splicing quite substantially. At the very least, we can conclude that targeting the PTM to a region near the reactive splice site can lead to high levels of trans-splicing.

To dissect whether spacer length was important for efficient trans-splicing, we made two comparisons. Firstly, we compared CF-PTM50 with a long and short spacer (60 bases versus 10 bases) and showed that there was a $7 \%$ difference in trans-splicing between the two $(n=6$; data not shown), and secondly, we showed that the difference between CF-PTM50 and CF-PTM53 was also very similar whether the spacer was long or short $(n=7$, ninefold difference; data not shown). Thus, it appears that in this study, the spacer length (in the range that we tested) did not alter trans-splicing significantly.

\section{The presence of an intron in the PTM increases trans-splicing}

For 5'ER to work efficiently, the donor site of the PTM must be efficiently recognized by the splicing machinery. For internal exons, the close proximity of the splice sites that flank an exon are thought to help in exon definition (Berget 1995). Because 5'ER PTMs typically contain a large coding unit followed by a single splice site, we hypothesized that the recognition of this donor site is weak. To test this hypothesis, we introduced two changes to the PTM as follows: (1) we inserted a CFTR mini-intron 9 into the coding unit close to the PTM donor site (to create two exons of 1788 and 124 bp) (Fig. 5), and (2) we placed an IAS1 sequence element in the trans-splicing domain just downstream of the PTM donor. The latter element (IAS1) is a U-rich sequence that enhances the splicing of the alternative exon IIIb from the fibroblast growth factor receptor 2 gene (Del Gatto-Konczak et al. 2000). The presence of an IAS1 sequence (inserted at position +12 in the trans-splicing domain) produced no significant increase in activity (data not shown). The CFTR mini-intron sequence may serve two functions. Firstly, it may improve exon definition, and secondly, it may serve to increase the level of protein product by mechanisms other than splicing, such as increased mRNA 

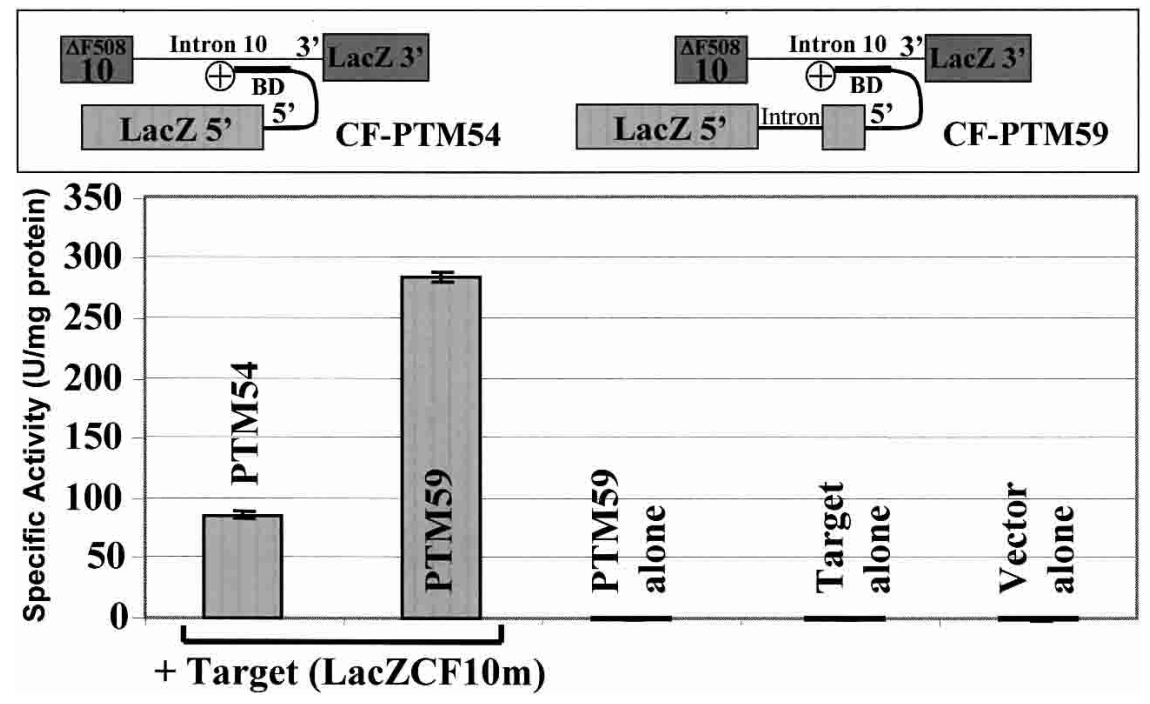

FIGURE 5. Inserting an intron in the PTM coding unit results in a significant improvement in trans-splicing efficiency. The two PTMs shown in the panel above the histogram are identical, except that CF-PTM59 has a 543-bp CFTR mini-intron 9 in the LacZ coding unit. The $3^{\prime}$ LacZ exon in CF-PTM59 is 124 bp long. Values are mean \pm standard error of two separate experiments. (BD) Binding domain.

processing, nucleo-cytoplasmic transport, and translation (Lu and Cullen 2003; Nott et al. 2003). In cotransfection experiments, PTMs containing a mini-intron gave a 3.4fold increase in $\beta$-galactosidase activity (Fig. 5, cf. CFPTM54 and CF-PTM59). In absolute units of $\beta$-galactosidase activity, the 199-mU/mg protein was the highest observed and represented a 65 -fold increase over that obtained with the original PTM (CF-PTM42).

\section{DISCUSSION}

\section{5' Exon replacement}

This work is significant because it demonstrates that SMaRT can be used to efficiently reprogram the $5^{\prime}$ end of mRNA. This process, termed $5^{\prime}$ exon replacement ( $\left.5^{\prime} \mathrm{ER}\right)$, has many potential applications, including gene correction, imaging of living cells in vitro or in vivo, or suicide gene therapy. We have shown that (1) trans-splicing by SMaRT can be performed with coding sequences of at least $1.9 \mathrm{~kb}$; (2) trans-splicing is precise; (3) trans-splicing can be achieved by tethering the PTM to the target in several different locations; (4) the efficiency can be increased many fold (65-fold in this study); and (5) functional $\beta$-galactosidase and CFTR ion channels can be generated in human cells.

Previous work using SMaRT focused mainly on the application of $3^{\prime} \mathrm{ER}$, in which PTMs were engineered to replace the $3^{\prime}$ end of target mRNA (Puttaraju et al. 1999, 2001; Mansfield et al. 2000; Liu et al. 2002). Each form of SMaRT has its own specific design features and potential advantages (Garcia-Blanco et al. 2000). As is true for all forms of mRNA repair, each PTM need only deliver a fraction of the full-length coding sequence used in the conventional approaches for RNA or gene therapy. However, PTMs that perform $3^{\prime}$ ER should not possess a methionine initiator codon, which they acquire from the target by trans-splicing. However, 5'ER PTMs do require the methionine codon for expression. Another difference is that $5^{\prime}$ ER PTMs can be modified at their $5^{\prime}$ end for the purpose of increasing translation, whereas mRNA modified by $3^{\prime}$ ER PTMs will acquire the endogenous target $5^{\prime}$ UTR. Conversely, mRNA modified by $5^{\prime} \mathrm{ER}$ will contain the endogenous $3^{\prime}$ UTR and poly(A) signal sequence, whereas $3^{\prime} \mathrm{ER}$ PTMs can benefit by inclusion of a specialized poly(A) signal and 3' UTR in the construct, which can significantly influence mRNA processing, stability, and transport. However, both modes of repair confer important advantages in the reduction of transgene size, which allows efficient packaging of PTMs into vector delivery systems with limited packaging capacity, for example, AAV, the ability to generate mRNA of normal length, and a much broader choice of regulatory sequences. A second and profound advantage of SMaRT, in general, is the acquisition of endogenous regulation, as the level of the mutant target is controlled by the natural transcriptional machinery. The fact that SMaRT can reprogram mRNA at either the $5^{\prime}$ or $3^{\prime}$ end suggests that any codon within a single mRNA can be modified regardless of position. This significantly broadens the applications of SMaRT and provides a choice of how to repair particular mRNA.

\section{5' Exon replacement is highly dynamic}

This study provided information on design features that may be generally applicable to SMaRT. Two important findings were (1) efficient trans-splicing can be achieved by tethering the PTM at many different locations in a premRNA, and (2) a few changes in PTM design could have a profound effect on the level of trans-splicing. The inclusion of a mini-intron in the PTM-coding region, and retargeting of the PTM close to the target acceptor site, resulted in a 65 -fold increase in trans-splicing. The former design feature has also been tested in $3^{\prime}$ ER constructs, and resulted in a substantial increase in reporter activity (S.A. Mansfield, R. Hawkins-Clark, M. Puttaraju, L.A. Mitchell, and M.A. Garcia-Blanco, unpubl.). It has been reported previously that including an intron in a cDNA transgene or reporter gene can have a profound impact on protein expression in vitro 
in cells and in vivo in animal models (Choi et al. 1991; Whitelaw et al. 1991; Zielere and Huynh 2002). The reasons for this are largely unknown and may differ between different cell types and constructs, and the context of the intron insertion (Nott et al. 2003). Certainly, the effect does not seem to be merely a simple requirement for cis-splicing (Whitelaw et al. 1991). Several studies suggest that the process of cis-splicing itself results in the deposition of protein factors on the mRNA, referred to as the exon junction complex (LeHir et al. 2000) that may subsequently affect downstream events such as mRNA transport and translation (for review, see Maniatis and Reed 2002). Some reports also indicate that splicing can substantially impact the downstream process of $3^{\prime}$ end processing and cleavage (Niwa et al. 1990; Lu and Cullen 2003).

Another design feature that significantly affected transsplicing was retargeting of the PTM close to the target acceptor. In the case of $3^{\prime}$ ER PTMs, we have shown recently that targeting a PTM to the $5^{\prime}$ end of a large intron $(14 \mathrm{~kb})$ as opposed to the $3^{\prime}$ end resulted in a 10 -fold difference (S.A. Mansfield, P. Du, S. Hiriyanna, R. Bartel, and E. Otto, unpubl.). In that case, this difference may be explained by the (temporal) kinetic advantage gained by targeting a PTM to the region of the intron that is first transcribed, that is, the donor site, when the target acceptor site has not been synthesized. However, in the case of trans-splicing by $5^{\prime} \mathrm{ER}$, the target acceptor site has to be transcribed before either cis- or trans-splicing can be initiated, and so there is no kinetic advantage gained by having the PTM close to the target acceptor site. The reason here may be fundamentally different. The elongating RNA polymerase II (RNAP II) is thought to interact, via the phosphorylated carboxy-terminal domain of the largest subunit with splicing factors, and these interactions may sequester exons near the polymerase (Morris and Greanleaf 2000; Goldstrohm et al. 2001). In very long introns, which may loop away from the elongating RNAP II, there will be regions adjacent to the exons, but not sequestered by the transcriptional machinery that may be optimal for trans-splicing. In contrast, the intronic regions immediately adjacent to the splice sites may be sterically hindered by the transcriptional machinery.

The demonstration that mRNA can be completely rewritten by $5^{\prime}$ ER using SMaRT and that the efficiency of transsplicing can be increased greatly by subtle changes in PTM structure, demonstrates the dynamic nature of SMaRT. The ability to efficiently perform $5^{\prime} \mathrm{ER}$ significantly broadens the applications of SMaRT technology.

\section{MATERIALS AND METHODS}

\section{Plasmid construction}

All PCR products were generated with either REDTaq (Sigma) or cloned Pfu (Stratagene) DNA Polymerase. Target CFTR-T11 (see Fig. 1A) was assembled from two separate PCR fragments, both amplified from an existing plasmid template. PCR primers for amplification contained restriction sites for directed cloning (NheI and ApaI, and ApaI and NotI). PCR products were digested with the appropriate restriction enzymes and cloned into the mammalian expression plasmid pc3.1DNA(-) (Invitrogen) using NheI, ApaI, and Not I sites. Exon 10 was also engineered with two stop codons immediately downstream of a group of three methionines at the $5^{\prime}$ end of exon 10 to ensure that the target could not be translated into a fragment of CFTR. The trans-splicing domains of each PTM were created by annealing different sets of oligonucleotides and ligating them into the appropriate PTM vector using AgeI, HindIII, and EcoRI restriction sites. Oligodeoxynucleotide primers were procured from Sigma Genosys. For the PTMs, the sequence between the putative transcription start site and the $5^{\prime}$ cloning site NheI is TAGAGAACCCACTGCTTACTGGCTTATCGAAATTAAT ACGACTCACTATAGGGAGACCCAAGCTG. For the targets, the sequence up to the XhoI cloning site is TAGAGAACCCACTGCT TACTGGCTTATCGAAATTAATACGACTCACTATAGGGAGAC CCAAGCTGGCTAGCGTTTAAACGGGCCCTCTAGA.

\section{DNA sequencing}

DNA templates were sequenced at the UNC-CH Automated DNA Sequencing Facility on a model 373A DNA Sequencer (Applied Biosystems) using a Taq Dideoxy Terminator Cycle Sequencing Kit (Applied Biosystems) and 10 pmole of primer per reaction.

\section{Cell culture and transfections}

Constructs were cotransfected in human embryonic kidney (HEK) 293 T or 293 cells $\left(1.5 \times 10^{6}\right.$ cells per $60-\mathrm{mm}$ poly-d-lysine-coated dish) using LipofectaminePlus (Invitrogen), and the cells were harvested $48 \mathrm{~h}$ after the start of transfection. HEK 293T cells were grown in Dulbecco's Modified Eagle's Medium (Invitrogen) supplemented with $10 \% \mathrm{v} / \mathrm{v}$ fetal bovine serum (Hyclone). All cells were kept in a humidified incubator at $37^{\circ} \mathrm{C}$ and $5 \% \mathrm{CO}_{2}$.

\section{RT-PCR}

Total RNA was isolated using a MasterPure RNA isolation kit (Epicenter Technologies) and used in RT-PCR (by use of an EZRT-PCR kit; Applied Biosystems) as described previously (Puttaraju et al. 1999). Each reaction contained $250 \mathrm{ng}$ of total RNA and $120 \mathrm{ng}$ of a $5^{\prime}$ and $3^{\prime}$ specific primer in a $60-\mu \mathrm{L}$ reaction volume. RT-PCR products were electrophoresed on $2 \%$ SeaKem agarose gels. The oligonucleotide primers used to generate cis- and trans-spliced products are 5'-GACCTCTGCAGACTTCACTTC TAATGATGATTATGG-3' (CF1), 5'-CGCTGGAAAAACGAGC TTGTTG-3' (CF93), and 5'-ACTCAGTGTGATTCCACCTTCTC $3^{\prime}(\mathrm{CF} 111)$

\section{$\beta$-galactosidase in-solution assay and in situ staining}

Total cellular protein from cells transfected with expression plasmids was isolated by freeze thaw method and assayed for $\beta$-galactosidase activity using a $\beta$-gal assay kit (Invitrogen). Protein concentration was measured by the dye-binding assay using BioRad protein assay reagents (BIO-RAD). Cells were monitored for 
the expression of functional $\beta$-galactosidase using a $\beta$-gal staining kit (Invitrogen).

\section{lodide efflux assays}

Anion efflux was measured by loading cell monolayers with ${ }^{125} \mathrm{Io}-$ dine as described previously (Venglarik et al. 1990; Strong et al. 1993). The baseline efflux rate was determined during two collection periods ( $2 \mathrm{~min}$ each); cells were then stimulated with $10 \mathrm{M}$ forskolin plus $5 \mathrm{mM}$ IBMX, and the cAMP-stimulated efflux rate was measured during three additional collection periods $(3 \mathrm{~min}$ each).

\section{ACKNOWLEDGMENTS}

This work was supported by grants from the National Institutes of Health (I R43 DK56526-03) and the Cystic Fibrosis Foundation (MANSFI00GO). We thank Carl Baker (NCI) for his critical reading of the manuscript.

The publication costs of this article were defrayed in part by payment of page charges. This article must therefore be hereby marked "advertisement" in accordance with 18 USC section 1734 solely to indicate this fact.

Received June 6, 2003; accepted July 8, 2003.

\section{REFERENCES}

Berget, S.M. 1995. Exon recognition in vertebrate splicing. J. Biol. Chem. 270: 2411-2414.

Chao, H., Mansfield, S.G., Bartel, R.C., Hiriyanna, S., Mitchell, L.G., Garcia-Blanco, M.A., and Walsh, C.E. 2003. Phenotype correction of hemophilia A mice by spliceosome-mediated RNA trans-splicing. Nat. Med. 9: 1015-1019.

Choi, T., Huang, M., Gorman, C., and Jaenisch, R. 1991. A generic intron increases gene expression in transgenic mice. Mol. Cell. Biol. 11: 3070-3074.

Chowrira, B.M., Pavco, P.A., and McSwiggen, J.A. 1994. In vitro and in vivo comparison of hammerhead, hairpin, and hepatitis $\delta$ virus self-processing ribozyme cassettes. J. Biol. Chem. 269: 2585625864.

Del Gatto-Konczak, F., Bourgeois, C.F., Le Guiner, C., Kister, L., Gesnel, M.C., Stevenin, J., and Breathnach, R. 2000. The RNA-binding protein TIA-1 is a novel mammalian splicing regulator acting through intron sequences adjacent to a $5^{\prime}$ splice site. Mol. Cell. Biol. 20: 6287-6299.

Garcia-Blanco, M.A., Puttaraju, M., Mansfield, S.G., and Mitchell, L.G. 2000. Spliceosome-mediated RNA trans-splicing in gene therapy and genomics. Gene Ther. Reg. 1: 141-163.

Goldstrohm, A.C., Greenleaf, A.L., and Garcia-Blanco, M.A. 2001. Co-transcriptional splicing of pre-messenger RNAs: Considerations for the mechanism of alternative splicing. Gene 277: 31-47.

Jobling, S.A. and Gehrke, L. 1987. Enhanced translation of chimeric messenger RNAs containing a plant viral untranslated leader sequence. Nature 325: 622-625.

Krause, M. and Hirsh, D.A. 1987. trans-spliced leader sequence on actin mRNA in C. elegans. Cell 49: 753-761.

Lan, N., Howrey, R.P., Lee, S.W., Smith, C.A., and Sullenger, B.A.
1998. Ribozyme-mediated repair of sickle $\beta$-globin mRNAs in erythrocyte precursors. Science 280: 1593-1596.

Le Hir, H., Moore, M.J., and Maquat, L.E. 2000. Pre-mRNA splicing alters mRNP composition: Evidence for stable association of proteins at exon-exon junctions. Genes \& Dev. 14: 1098-1108.

Liu, X., Jiang, Q., Mansfield, S.G., Puttaraju, M., Zhang, Y., Zhou, W., Cohn, J.A., Garcia-Blanco, M.A., Mitchell, L.G., and Engelhardt, J.F. 2002. Partial correction of endogenous $\Delta$ F508 CFTR in human cystic fibrosis airway epithelia by spliceosome-mediated RNA trans-splicing. Nat. Biotech. 20: 47-52.

Lu, S. and Cullen, B.R. 2003. Analysis of the stimulatory effect of splicing on mRNA production and utilization in mammalian cells. RNA 9: 618-630.

Maniatis, T. and Reed, R. 2002. An extensive network of coupling among gene expression machines. Nature 416: 499-506.

Mansfield, S.G., Kole, J., Puttaraju, M., Yang, C.C., Garcia-Blanco, M.A., Cohn, J.A., and Mitchell, L.G. 2000. Repair of CFTR mRNA by spliceosome-mediated RNA trans-splicing. Gene Ther. 7: 18851895.

Moore, M.J., Query, C.C., and Sharp, P.A. 1999. Splicing of precursors to mRNA by the spliceosome. In The RNA world (eds. R.F. Gesteland and J.F. Atkins), pp. 303-357. Cold Spring Harbor Laboratory Press, Cold Spring Harbor, NY.

Morris, D.P. and Greenleaf, A.L. 2000. The splicing factor, Prp40, binds the phosphorylated carboxyl-terminal domain of RNA polymerase II. J. Biol. Chem. 22: 39935-39943.

Niwa, M., Rose, S.D., and Berget, S.M. 1990. In vitro polyadenylation is stimulated by the presence of an upstream intron. Genes \& Dev. 4: 1552-1559.

Nott, A., Meislein, S.H., and Moore, M.J. 2003. A quantitative analysis of intron effects on mammalian gene expression. RNA 9: 607-617.

Puttaraju, M., Jamison, S.F., Mansfield, S.G., Garcia-Blanco, M.A., and Mitchell, L.G. 1999. Spliceosome-mediated RNA trans-splicing as a tool for gene therapy. Nat. Biotech. 17: 246-252.

Puttaraju, M., DiPasquale, J., Baker, C.C., Mitchell, L.G., and GarciaBlanco, M.A. 2001. Messenger RNA repair and restoration of protein function by spliceosome-mediated RNA trans-splicing. Mol. Ther. 4: 105-114.

Rogers, C.S., Vanoye, C.G., Sullenger, B.A., and George, A.L. 2002. Functional repair of a mutant chloride channel using a trans-splicing ribozyme. J. Clin. Invest. 110: 1783-1789.

Shi, Y. 2003. Mammalian RNAi for the masses. Trends Genet. 19: 9-12. Strong, D.J., Wilkinson, D.J., Mansoura, M.K., Henze, K., Yang, Y., Wilson, J.M., Cohn, J.A., Dawson, D.C., Frizzell, R.A., and Collins, F.S. 1993. Expression of an abundant alternatively spliced form of the cystic fibrosis transmembrane conductance regulator (CFTR) gene is not associated with a cAMP-activated chloride conductance. Hum. Mol. Genet. 2: 225-230.

Sullenger, B.A. and Gilboa, E. 2002. Emerging clinical applications of RNA. Nature 418: 252-258.

Van der Ploeg, L.H. 1986. Discontinuous transcription and splicing in trypanosomes. Cell 47: 479-480.

Venglarik, C.J., Bridges, R.J., and Frizzell, R.A. 1990. A simple assay for agonist-regulated $\mathrm{Cl}$ and $\mathrm{K}$ conductances in salt-secreting epithelial cells. Am. J. Physiol. 259: C358-C364.

Watanabe, T. and Sullenger, B.A. 2000. Induction of wild-type p53 activity in human cancer cells by ribozymes that repair mutant p53 transcripts. Proc. Natl. Acad. Sci. 97: 8490-8494.

Whitelaw, C.B., Archibald, A.L., Harris, S., McClenaghan, M., Simons, J.P., and Clark, A.J. 1991. Targeting expression to the mammary gland: Intronic sequences can enhance the efficiency of gene expression in transgenic mice. Transgenic Res. 1:3-13.

Zieler, H. and Huynh, C.Q. 2002. Intron-dependent stimulation of marker gene expression in cultured insect cells. Insect Mol. Biol. 11: 87-95. 

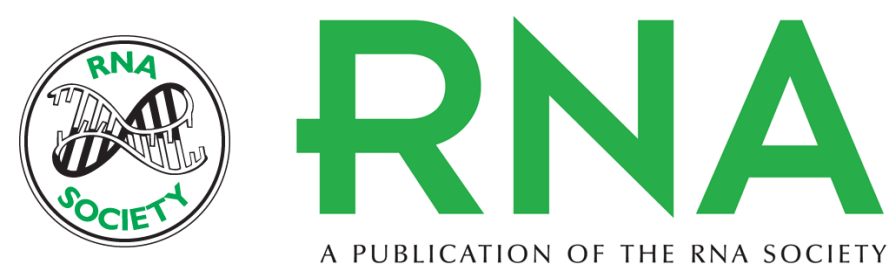

A PUBLICATION OF THE RNA SOCIETY

\title{
5' Exon replacement and repair by spliceosome-mediated RNA trans -splicing
}

\author{
S. GARY MANSFIELD, REBECCA HAWKINS CLARK, M. PUTTARAJU, et al.
}

RNA 2003 9: 1290-1297

$\begin{array}{ll}\text { References } & \begin{array}{l}\text { This article cites } 27 \text { articles, } 10 \text { of which can be accessed free at: } \\ \text { http://rnajournal.cshlp.org/content/9/10/1290.full.html\#ref-list-1 }\end{array}\end{array}$

License

Email Alerting Receive free email alerts when new articles cite this article - sign up in the box at the Service top right corner of the article or click here. 